\title{
Simultaneous oral administration of Salmonella Infantis and S. Typhimurium in chicks
}

\author{
Koichi Murakami ${ }^{1,2^{*}}$, Eriko Maeda-Mitani ${ }^{2}$, Daisuke Onozuka ${ }^{3}$,Tamie Noda ${ }^{2,6}$, Nobuyuki Sera ${ }^{2}$, Hirokazu Kimura ${ }^{1}$, \\ Shuji Fujimoto ${ }^{4}$ and Satoshi Murakami ${ }^{5}$
}

\begin{abstract}
Background: To confirm the hypothesis that Salmonella enterica subspecies enterica serovar (S.) Infantis has higher basic reproductive rates in chicks compared with other Salmonella serovars, 1-day-old specific-pathogen-free chicks $(n=8)$ were challenged simultaneously with S. Infantis and S. Typhimurium per os. Challenged chicks (Group A) were then housed with non-infected chicks (Group B, $n=4$ ) for 6 days (from 2 to 8 days of age). Group B birds were then housed with other non-infected birds (Group $C, n=4$ ), which were then transferred to cages containing a further group of untreated chicks (Group $D, n=2$ ). A control group consisting of four non-infected chicks was used for comparison. All chickens were humanely sacrificed at 18 days of age, and Salmonella from bowel and liver samples were enumerated.
\end{abstract}

Results: Both serovars were isolated from all groups except the control group. S. Typhimurium was isolated at a greater frequency than $S$. Infantis from the bowel samples of chicks from Groups B, C and D, while no differences in colonisation rates were observed between the two serovars in liver samples from Groups B, C and D. S. Typhimurium, but not S. Infantis, was immunohistochemically detected in the lamina propria of the cecum and rectum in five birds of Group A. Despite the competitive administration, neither of the two serovars completely excluded the other, and no differences were observed in basic reproductive rates between the two serovars.

Conclusions: These findings, together with data from previous studies, suggest that the initial quantitative domination of S. Infantis in chicken flocks may explain why this serovar is predominant in broiler chickens.

Keywords: Salmonella infantis, Salmonella typhimurium, Chicken, Basic reproductive rate, Oral administration, Chick bowel

\section{Background}

Human infections caused by ingestion of chicken meat contaminated with Salmonella enterica subsp. enterica serovar $(S$.) Infantis are a significant public health concern in many countries, including Japan $[1,2]$. Salmonellosis caused by non-typhoidal Salmonella serovars occurs fairly frequently worldwide [3]. S. Infantis is a major non-typhoidal Salmonella serovar in Japan, and is the predominant Salmonella contaminant of chicken

\footnotetext{
* Correspondence: kmuraka@nih.go.jp

1 Infectious Disease Surveillance Center, National Institute of Infectious

Diseases, 4-7-1 Gakuen, Musashi-murayama, Tokyo 208-0011, Japan

${ }^{2}$ Fukuoka Institute of Health and Environmental Sciences, Mukaizano 39,

Dazaifu, Fukuoka 818-0135, Japan

Full list of author information is available at the end of the article
}

meat. It was found in more than $23 \%$ of retail chicken meat samples from Fukuoka Prefecture, Kyushu, Japan [1], and human salmonellosis cases caused by $S$. Infantis-contaminated chicken meat are relatively frequent in Kyushu [4,5]. S. Infantis is also the dominant serovar in broiler farms in western Japan [6], although why and how it became the dominant serovar remains unresolved.

We hypothesised that $S$. Infantis may infect susceptible chickens at a higher frequency than other serovars, perhaps because of a higher basic reproductive rate in chickens [7]. However, little is known about the issue. Several studies have administered multiple Salmonella serovars at different intervals (1 day or more) in an 
attempt to understand the dynamics of infection $[8,9]$, but simultaneous administration of multiple Salmonella serovars is rare. Therefore, in the current study, we simultaneously infected 1-day-old chicks with $S$. Infantis and $S$. Typhimurium, and then housed the infected birds with non-infected chicks. The aim of the study was to determine whether $S$. Infantis more frequently passes from infected to non-infected chicks than $S$. Typhimurium.

\section{Methods}

\section{Salmonella strains and chickens}

S. Infantis strains 200-1, 1582 and 1596, isolated in 1995, 2005 and 2004, respectively, from chicken meat and broilers in western Japan, were used in the current study. All three strains belonged to the most dominant genotype, pulsed-field profile 4 , as determined by pulsed-field gel electrophoresis analysis [10]. The three S. Typhimurium strains, 586, R6 and R38, were isolated from beef and humans in 2005, 1999 and 1999, respectively. All strains were stored at $-80{ }^{\circ} \mathrm{C}$.

Specific-pathogen-free (SPF) layer chickens (L-M line) were purchased from Nisseiken (Oume, Japan). At 0 days old, chicks were transported from Tokyo to Dazaifu by plane and car. Radiation-sterilised food (Funabashi Farm Co., Funabashi, Japan) and tap water were provided ad libitum, and sterilised bedding (Oriental Yeast Co., Tokyo, Japan) was used. Two to four birds were housed in each sterilised cage (Allentown, Allentown, NJ, USA), and cages were placed in a low-atmospheric-pressure caring apparatus $(-10 \mathrm{hPa}$ compared with room atmospheric pressure), which allowed for adjustments to temperature and humidity (Natsume Sesakusyo Co., Tokyo, Japan). Chicks were transferred to freshly autoclaved cages every 2 days during the experimental period. The temperature and humidity were initially set at $33{ }^{\circ} \mathrm{C}$ and $75 \%$, respectively. These were decreased by $0.5{ }^{\circ} \mathrm{C}$ and $0.5 \%$, respectively, per day, to achieve final conditions of $28.5{ }^{\circ} \mathrm{C}$ and $71.5 \%$, respectively. The animal room was controlled with a $12 \mathrm{~h}$ light/dark cycle.

\section{Salmonella administration}

Frozen $\left(-80{ }^{\circ} \mathrm{C}\right)$ aliquots of each of the Salmonella strain stocks were inoculated into 3-ml volumes of LuriaBertani (LB) broth (Becton Dickinson, Franklin Lakes, NJ, USA) and incubated with continuous shaking at $35^{\circ}$ $\mathrm{C}$ for about $18 \mathrm{~h}$. The overnight bacterial cultures were then diluted with LB broth heated to $42{ }^{\circ} \mathrm{C}$. The dilution volumes were determined by a preliminary dose-finding experiment (data not shown). Equal volumes of the three cultures of each serovar were mixed, and a $0.3-\mathrm{ml}$ aliquot of the pooled cocktail of $S$. Typhimurium was administered into the crop of eight 1-day-old chicks using syringes with gavage needles. A $0.3-\mathrm{ml}$ aliquot of $S$.
Infantis cocktail was then immediately administered to the same chicks. Bacterial cell counts were carried out for each of the cocktails following administration, and showed that the $0.3-\mathrm{ml}$ aliquots of $S$. Typhimurium and $S$. Infantis contained $2.7 \times 10^{6}$ and $3.1 \times 10^{6}$ colonyforming units, respectively.

\section{Caging design}

Figure 1 shows the caging schedule of the inoculated birds (seeder birds) with the non-infected birds (recipients). On day 2 post-inoculation (2 days old), the seeder birds (Group A, $n=8$ ) were caged with the first group of recipients (Group B, $n=4$ ). On day 8, Group B was caged with the second group of recipients (Group C, $n=4)$. Group $C$ was then caged with the third recipient group (Group D, $n=2$ ) on day 15. Control group birds $(n=4)$ were caged by themselves without any exposure to the Salmonella strains. All experimental animals were sacrificed by exsanguination under carbon dioxide gasanaesthesia at day 18.

\section{Enumeration of Salmonella from chick samples}

Bowels and livers were dissected from the euthanised animals and then minced using sterilised scissors. The minced samples were then homogenised with 9 volumes of sterile saline using a Stomacher paddle blender (Seward, Worthing, UK). Ten-fold serial dilutions of the homogenised solutions were carried out, and $0.1 \mathrm{ml}$ of each dilution was plated on Salmonella-Shigella (SS) agar (Eiken Chemical Co., Tokyo, Japan) in duplicate and incubated at $35{ }^{\circ} \mathrm{C}$. SS agar was used on the basis of a preliminary agar selection test that showed similar growth support for both serovars. Following incubation for 2 days, Salmonella colonies were counted. Thirty isolates from each sample were identified as $S$. Typhimurium or $S$. Infantis using somatic $(\mathrm{O})$ antisera $\mathrm{O} 4$ and $\mathrm{O}$, respectively (Denka Seiken Co., Tokyo, Japan). Statistical analyses were carried out using the chi-square test.

\section{Histopathology and immunohistochemistry}

Five chickens from Group A were sacrificed at day 5 and examined using histopathology and immunohistochemistry. Chicken bowels were fixed with $20 \%$ formalin, and embedded in paraffin wax. Sections (3$4 \mu \mathrm{m}$ thick) were then cut and stained with haematoxylin and eosin. Sections of the cecum, rectum and bursa of Fabricius were used for the detection of Salmonella serovar O4- and O7-group antigens. Sections were immunostained using the streptavidin-biotinperoxidase conjugate (SAB) method, as previously described [11]. Controls for the SAB method were performed by omitting the primary antisera. 


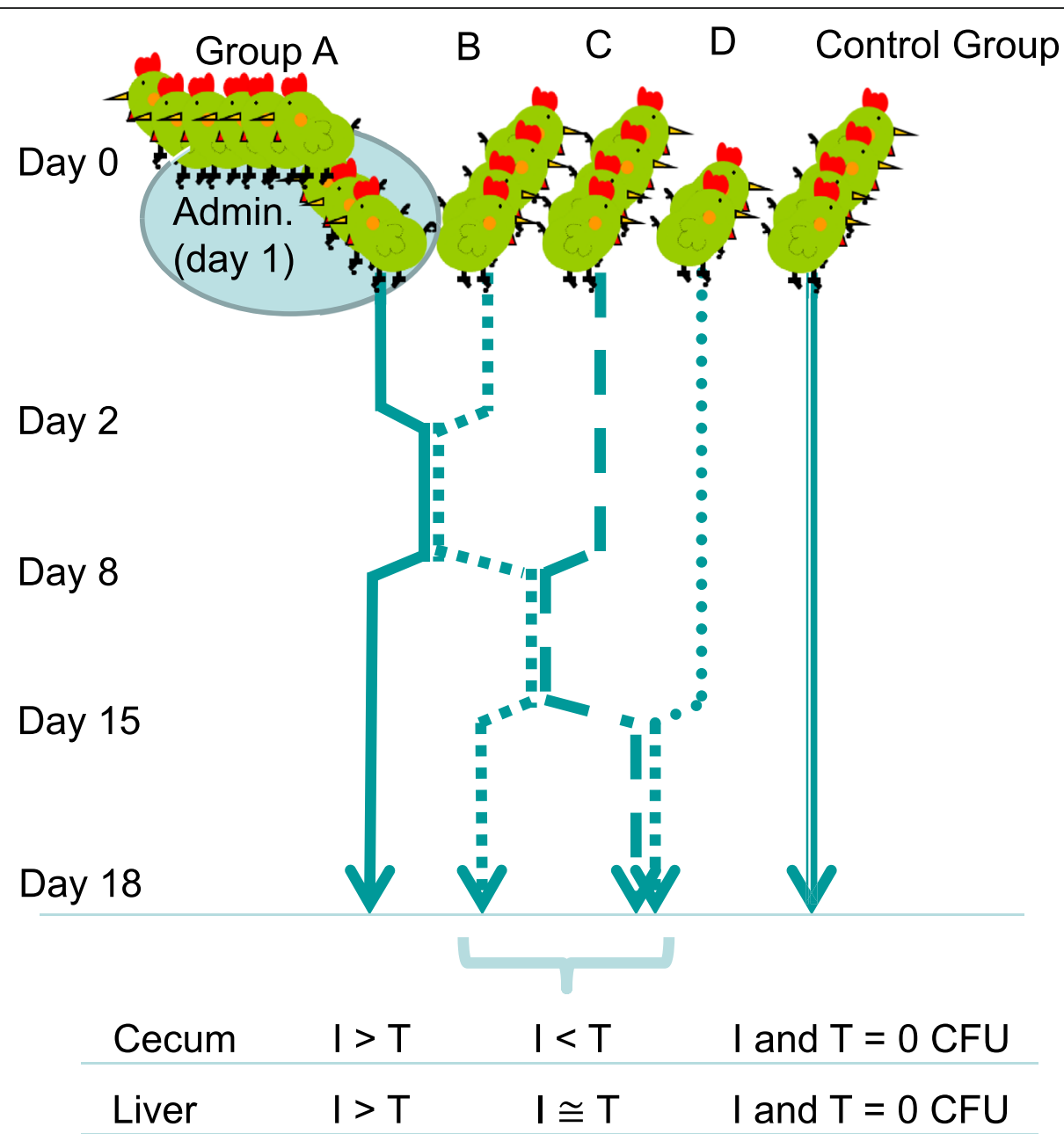

Fig. 1 Experimental procedure for Salmonella administration. Eight 1-day-old birds were simultaneously administered with Salmonella enterica subspecies enterica serovar Infantis and S. Typhimurium (Group a), and then housed in the same cage as four non-infected birds (Group b) on days 2-8. Group b birds were then caged with Group $\mathbf{c}$ birds (four non-infected birds) on days 8-15. Group $\mathbf{c}$ birds were then housed with a final group of two non-infected birds (Group d) for days 15-18. I and T denote S. Infantis and S. Typhimurium, respectively. Five of eight birds were sacrificed at day 5 and examined using histopathology and immunohistochemistry

\section{Results}

\section{Colonisation of chicks}

Both Salmonella serovars were isolated from all samples collected from all birds, except for the control group. Levels of $S$. Infantis colonisation were significantly greater than those of $S$. Typhimurium in the bowel samples of Group A birds $(P<0.001)$ (Table 1). In contrast, the bowel samples of recipient birds (Groups B-D) showed significantly higher levels of $S$. Typhimurium colonisation compared with those of $S$. Infantis $(P<0.001$; chi-square test). $S$. Infantis was also significantly more prevalent than $S$. Typhimurium in the liver samples of Group A birds $(P<0.001)$, whereas there was no difference in colonisation rates between the serovars in any of the liver samples from recipient birds (Groups B-D).

Mean body weights (in $\mathrm{g}$ ) at day 1 were as follows: Group A, $36.8 \pm 4.0$; Group B, $41.3 \pm 1.2$; Group C,
$38.1 \pm 1.4$; Group D, $46.0 \pm 0$; control group, $41.3 \pm 4.4$. Mean body weights (in g) at day 18 were as follows: Group A, $154.5 \pm 7.3$; Group B, $177.3 \pm 16.2$; Group C, $167.9 \pm 4.5$; Group D, $207.5 \pm 1.5$; control group, $177.8 \pm 12.4$.

\section{Histopathology}

Although there were no macroscopic lesions observed in the intestines of chicks administered with both Salmonella serovars, a number of instances of heterophil infiltration were observed in the epithelial layer and lamina propria of the cecum (Fig. 2a) and rectum. The lymphoid follicles of the bursa of Fabricius also had a "starry-sky" appearance.

\section{Immunohistochemistry}

Several Salmonella serovar O4 antigens, indicating $S$. Typhimurium, and $\mathrm{O} 7$ antigens, indicating $S$. Infantis, 
Table 1 Salmonella enterica subsp. enterica serovar (S.) Infantis and S.Typhimurium isolation rates at 18 days post administration of 1day-old chicks

\begin{tabular}{llllll}
\hline Organ & Group & Number of chicks & Salmonella colony count Mean \pm SD (CFU / g) & S. Infantis \% & Description \\
\hline Bowels & A & 3 & $1.6 \times 10^{7} \pm 0.6 \times 10^{7}$ & $69 \pm 11 \%$ & More S. Infantis was isolated $^{*}$ \\
& B & 4 & $8.5 \times 10^{6} \pm 3.5 \times 10^{6}$ & $40 \pm 7 \%$ & More S. Typhimurium was isolated $^{*}$ \\
& C & 4 & $1.4 \times 10^{7} \pm 0.8 \times 10^{7}$ & $49 \pm 13 \%$ & More S. Typhimurium was isolated $^{*}$ \\
& D & 2 & $9.7 \times 10^{6} \pm 1.8 \times 10^{6}$ & $44 \pm 3 \%$ & More S. Typhimurium was isolated $^{*}$ \\
& Control & 4 & Not isolated & & More S. Infantis was isolated \\
Liver & A & 3 & $2.6 \times 10^{4} \pm 3.3 \times 10^{4}$ & $62 \pm 18 \%$ & No difference \\
& B & 4 & $9.9 \times 10^{3} \pm 5.5 \times 10^{3}$ & $68 \pm 13 \%$ & No difference \\
C & 4 & $1.4 \times 10^{4} \pm 1.9 \times 10^{4}$ & $53 \pm 28 \%$ & No difference \\
D & 2 & $2.2 \times 10^{4} \pm 3.0 \times 10^{4}$ & $35 \pm 7 \%$ & \\
Control & 4 & Not isolated & &
\end{tabular}

${ }^{*} P<0.001$

were detected in cecal and rectal contents using immunohistochemistry. Although there were no Salmonella serovar $\mathrm{O} 7$ antigens in the parenchyma of the cecum (Fig. 2b), rectum or bursa of Fabricius, O7 immunopositive signals were detected in cecal and rectal crypts. In addition, immuno-positive signals of Salmonella serovar $\mathrm{O} 4$ were detected in the lamina propria of the cecum (Fig. 2c) and rectum, as well as in lymphoid follicles of the bursa of Fabricius.

\section{Discussion}

This study produced three main findings. First, no difference was observed in basic reproductive rates between the two serovars. Second, neither of the two serovars completely excluded the other, despite their competitive administration. Finally, $S$. Infantis invasion rates of the lamina propria of the cecum and rectum were lower than those of $S$. Typhimurium, even in the inoculated birds (Group A).
The findings of the current study, together with previous data, may explain the dominance of $S$. Infantis in chicken meat. A study in which heterologous serovars of Salmonella were administrated to chicks at different intervals showed that the first strain to be inoculated inhibited the colonisation of the subsequent strains [9]. However, using simultaneous administration, we observed that the heterologous strains never inhibited each other in the inoculated chicks. Together, these findings suggest that the predominant Salmonella strain or serovar in a given environment (e.g. farm) may infect chicks and then inhibit colonisation by other strains or serovars. Subsequently, one dominant strain or serovar continuously maintains a higher colonisation rate in those chicken flocks compared with other strains or serovars. This may explain why $S$. Infantis is the dominant serovar in chicken meat in Japan.

Variations in the susceptibility of different chicken lines to Salmonella infection were reported in the

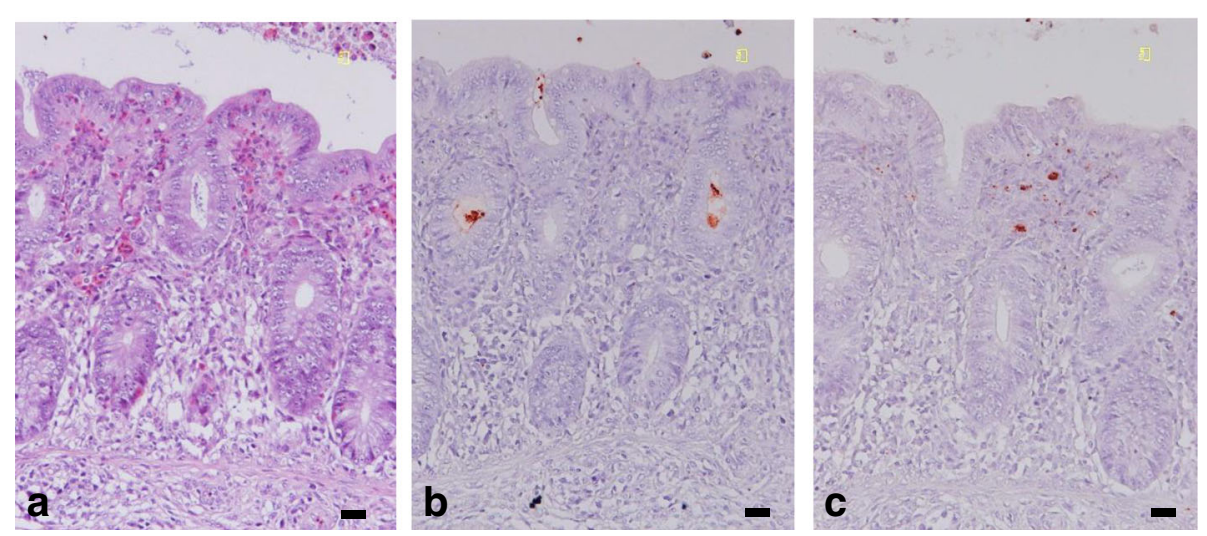

Fig. 2 Immunostaining of cecal contents from chicks on day 5 post simultaneous administration of Salmonella enterica subspecies enterica serovar Infantis and S. Typhimurium. (a) Haematoxylin and eosin staining showing infiltration of a number of heterophils into the epithelial layer and cecal Iamina propria. (b) Immuno-positive antigens against Salmonella serovar $\mathrm{O} 7$ were detected in cecal crypts. (c) Immuno-positive antigens against Salmonella serovar $\mathrm{O} 4$ were detected in the cecal lamina propria. White bars indicate $10 \mu \mathrm{m}$ 
middle of the twentieth century [12]. More recently, Leveque et al. (2003) reported differences in resistance to $S$. Typhimurium infection between chicken lines resulting from allelic variation in Toll-like receptor 4 [13]. Hu et al. (1997) also reported differences in Salmonella susceptibility among chicken lines based on Nramp1 (natural resistance-associated macrophage protein 1 ) and $T n c$ (a locus closely linked to $L p s$ ) variations [14]. Microbiota diversity in chicks can also affect susceptibility to infection [15]. However, little is known about differences in susceptibility to simultaneous inoculation of multiple Salmonella serovars in any chicken line. Therefore, while differences between chicken lines may affect susceptibility to Salmonella infection, in the current study, we focused on simultaneous infection with multiple Salmonella serovars. It would be interesting to carry out the same experiment in different chicken lines in the future to determine the effects of chicken line on susceptibility to simultaneous infection with multiple Salmonella serovars.

The simultaneous administration approach used in the current study produced different results from those described previously using individual administration of different Salmonella serovars [16]. Berndt et al. [16] reported that $S$. Infantis exhibited significantly lower invasion rates in the liver compared with $S$. Typhimurium after individual administration. In the present study, however, no differences were observed in the invasion rates of the liver between the two serovars. It is noteworthy that the two serovars never completely excluded each other in the liver after competitive administration. Non-detection of $S$. Infantis in the cecal lamina propria using immunohistochemistry may be the result of using sections from 5-day-old chicks. $S$. Infantis is less invasive of the cecal lamina propria at 5 days post-administration compared with at days 2 and 3 post-administration [9]. Moreover, a reduced ability to invade the cecal mucosa by $S$. Infantis compared with $S$. Typhimurium is consistent with the report by Berndt et al. [16].

\section{Conclusion}

The basic reproductive rates in chicks do not appear to differ between $S$. Infantis and $S$. Typhimurium. Moreover, neither of the serovars displayed a superior ability to colonise the chick bowel in comparison with the other. Therefore, the quantitative domination of $S$. Infantis in chicks, and the associated inhibition of subsequent colonisation by other Salmonella strains, may explain why $S$. Infantis is the predominant Salmonella serovar in chickens and chicken meat in Japan.

\section{Abbreviations}

LB: Luria-bertani; S.: Salmonella enterica subspecies enterica; SAB: Streptavidinbiotin-peroxidase conjugate; SPF: Specific-pathogen-free; SS: SalmonellaShigella

\section{Acknowledgements}

We are grateful to Dr. Katsuki, Mr. Oishi and Dr. Saeki from the Fukuoka Institute of Health and Environmental Sciences, Dazaifu, Japan, for their invaluable advice. We also thank Ms. Doi and Ms. Yamada of the National Institute of Infectious Diseases, Musashi-murayama, Japan, for their collaboration. We thank Tamsin Sheen, PhD, from Edanz Group (http://www.edanzediting.com) for editing a draft of this manuscript.

\section{Funding}

This work was supported by a grant from the Japanese Society for the Promotion of Science (KAKENHI; no. 15K08794), and the Research Program on Emerging and Re-emerging Infectious Diseases of the Japan Agency for Medical Research and Development, AMED (17fk0108106j0101).

\section{Availability of data and materials}

Data sharing not applicable to this article as no datasets were generated or analysed during the current study.

\section{Authors' contributions}

KM, EMM, DO, TN, SN and SM provided data, analysed the results and drafted the manuscript. HK and SF provided data, analysed the results and participated in revising the manuscript. All authors read and approved the final manuscript.

\section{Ethics approval and consent to participate}

This study was carried out in strict accordance with the guidelines of the Regulations for the Ethical and Humane Use of Experimental Animals at Fukuoka Institute of Health and Environmental Sciences, which is based on domestic standards, and approved by the Animal Ethics Committee of Fukuoka Institute of Health and Environmental Sciences under permit number $\mathrm{H} 241107$.

Consent for publication

Not applicable.

\section{Competing interests}

The authors declare that they have no conflicts of interest.

\section{Publisher's Note}

Springer Nature remains neutral with regard to jurisdictional claims in published maps and institutional affiliations.

\section{Author details \\ 'Infectious Disease Surveillance Center, National Institute of Infectious Diseases, 4-7-1 Gakuen, Musashi-murayama, Tokyo 208-0011, Japan. ${ }^{2}$ Fukuoka Institute of Health and Environmental Sciences, Mukaizano 39, Dazaifu, Fukuoka 818-0135, Japan. ${ }^{3}$ Department of Health Care Administration and Management, Kyushu University Graduate School of Medical Sciences, 3-1-1 Maidashi, Higashi-ku, Fukuoka 812-8582, Japan. ${ }^{4}$ Department of Health Sciences, Faculty of Medical Sciences, Kyushu University, 3-1-1 Maidashi, Higashi-ku, Fukuoka 812-8582, Japan. ${ }^{5}$ Department of Animal Science, Tokyo University of Agriculture, Atsugi, Kanagawa 243-0034, Japan. ${ }^{6}$ Present address: Kitachikugo Office for Health, Human Services, and Environmental Issues, 1642-1 Aikawa-machi Kurume, Fukuoka 839-0861, Japan.}

Received: 12 June 2017 Accepted: 23 August 2017

Published online: 31 August 2017

\section{References}

1. Noda T, Murakami K, Etoh Y, Okamoto F, Yatsuyanagi J, Sera N, Furuta M, Onozuka D, Oda T, Asai T, Fujimoto S. Increase in resistance to extendedspectrum cephalosporins in Salmonella isolated from retail chicken products in Japan. PLoS One. 2015;10:e0116927.

2. Hauser E, Tietze E, Helmuth R, Junker E, Prager R, Schroeter A, Rabsch W, Fruth A, Toboldt A, Malorny B. Clonal dissemination of Salmonella enterica serovar Infantis in Germany. Foodborne Pathog Dis. 2012;9:352-60.

3. Humphrey T. Public health aspects of Salmonella enterica in food production. In: Mastroeni P, Maskell D, editors. Salmonella infections, clinical, immunological and molecular aspects. Cambridge: Cambridge University Press; 2006. p. 89-116. 
4. Murakami K, Ishihara T, Horikawa K, Oda T. Features of Salmonella serovars among food handlers in Kyushu, Japan. New Microbiol. 2007;30:155-9.

5. Noda T, Murakami K, Ishiguro Y, Asai T. Chicken meat is an infection source of Salmonella serovar Infantis for humans in Japan. Foodborne Pathog Dis. 2010;7:727-35

6. Murakami K, Horikawa K, Ito T, Otsuki K. Environmental survey of salmonella and comparison of genotypic character with human isolates in western Japan. Epidemiol Infect. 2001;126:159-71.

7. Anderson RM. Analytical theory of epidemics. In: Krause RM, editor. Emerging infections. New York: Academic Press; 1998. p. 23-50.

8. Berchieri A Jr, Barrow PA. Further studies on the inhibition of colonization of the chicken alimentary tract with Salmonella typhimurium by precolonization with an avirulent mutant. Epidemiol Infect. 1990;104:427-41.

9. Methner U, Barrow PA, Berndt A. Induction of a homologous and heterologous invasion-inhibition effect after administration of Salmonella strains to newly hatched chicks. Vaccine. 2010;28:6958-63.

10. Murakami K, Horikawa K, Otsuki K. Genotypic characterization of human and environmental isolates of Salmonella choleraesuis subspecies choleraesuis serovar Infantis by pulsed-field gel electrophoresis. Microbiol Immunol. 1999:43:293-6.

11. Yokoyama E, Ando N, Ohta T, Kanada A, Shiwa Y, Ishige T, Murakami K, Kikuchi T, Murakami S. A novel subpopulation of Salmonella enterica serovar Infantis strains isolated from broiler chicken organs other than the gastrointestinal tract. Vet Microbiol. 2015;175:312-8.

12. Calenge $F$, Kaiser $P$, Vignal A, Beaumont $C$. Genetic control of resistance to salmonellosis and to Salmonella carrier-state in fowl: a review. Genet Sel Evol. 2010;42:11. https://doi.org/10.1186/1297-9686-42-11.

13. Leveque G, Forgetta V, Morroll S, Smith AL, Bumstead N, Barrow P, LoredoOsti JC, Morgan K, Malo D. Allelic variation in TLR4 is linked to susceptibility to Salmonella enterica serovar Typhimurium infection in chickens. Infect Immun. 2003;71:1116-24.

14. Hu J, Bumstead N, Barrow P, Sebastiani G, Olien L, Morgan K, Malo D. Resistance to salmonellosis in the chicken is linked to NRAMP1 and TNC. Genome Res. 1997;7:693-704

15. Mon KK, Saelao P, Halstead MM, Chanthavixay G, Chang HC, Garas L, Maga EA, Zhou H. Salmonella enterica serovars Enteritidis infection alters the indigenous microbiota diversity in young layer chicks. Front Vet Sci. 2015;2: 61. https://doi.org/10.3389/fvets.2015.00061.

16. Berndt A, Wilhelm A, Jugert C, Pieper J, Sachse K, Methner U. Chicken cecum immune response to Salmonella enterica serovars of different levels of invasiveness. Infect Immun. 2007;75:5993-6007.

\section{Submit your next manuscript to BioMed Central and we will help you at every step:}

- We accept pre-submission inquiries

- Our selector tool helps you to find the most relevant journal

- We provide round the clock customer support

- Convenient online submission

- Thorough peer review

- Inclusion in PubMed and all major indexing services

- Maximum visibility for your research

Submit your manuscript at www.biomedcentral.com/submit 